\title{
P-0464 - Effects of Tai Chi on physical and psychosocial outcomes among T2DM patients: a systematic review
}

\author{
YU, Xingfeng ${ }^{1}$; Chau, JPC $^{1}$; HUO, Lanting ${ }^{2}$
}

${ }^{1}$ The Nethersole School of Nursing, Faculty of Medicine, The Chinese University of Hong Kong; Hong Kong

${ }^{2}$ Faculty of Nursing, Health Science Center, Xi' an Jiaotong University, Xi'an, P. R. China

\section{Background}

The high prevalence and various complications of diabetes cast great burdens to patients, their families and the society [1]. Physical activity is an effective approach for type 2 diabetes (T2DM) patients to gain optimal control over the disease [2].

In recent years, Tai Chi is gaining popularity in DM management. Several reviews on the effectiveness of Tai Chi for T2DM patients were identified [3-5]. However, the findings of these reviews were not consistent. Besides, these reviews were conducted several years ago. With the rapid updates in this field, the findings may be out-of-date.

\section{Objective}

To synthesize and present the best available evidence on the effects of Tai Chi on physical and psychosocial outcomes among T2DM patients.

\section{Methods}

$>$ This systematical review was conducted in accordance with the PRISMA statements [6].

> Ten English or Chinese electronic databases, including MEDLINE, EMBASE, PsycINFO, CINAHL Complete, AMED and Cochrane Central Register of Controlled Trials, CNKI, Wan Fang, SinoMed and Airiti Library, were search through their inceptions to December 2016.

> This systematic review considered all journal articles reporting randomized controlled trials (RCTs) or controlled clinical trials (CCTs) that investigated the effects of Ba-Duan-Jin on physical or psychosocial outcomes, including haemoglobin A, glycosylated (HbAlc), fasting blood glucose (FBG), blood pressure, depression and quality of life (QOL), among adult T2DM patients.

$>$ Two independent reviewers selected studies according to the prespecified inclusion and exclusion criteria and appraised the risk of bias of the included studies. Discrepancies were solved by discussion or consulting to the third reviewer when consensus cannot be reached.

Dhen feasible, data were statistically pooled in a meta-analysis. Otherwise, narrative summaries were utilized.

\section{$\underline{\text { Results }}$}

- Eight studies (446 participants) were included in this systematic review. In general, the included studies were subject to substantial risk of bias. Seven studies utilized usual care control while the other one used sham exercise control.

- HbAlc was measured in six studies and the pooled analysis showed a favorable but non-significant effect on reducing HbAlc. However, after removing one study, of which the participants' average baseline $\mathrm{HbA1c}$ level was optimal $(<7.0 \%)$, the sensitivity analysis showed a statistically significant effect (MD: $-1.48 \%, 95 \%$ CI: $-2.58 \%$ to $-0.39 \%, \mathrm{p}<0.01$; Figure 1 ).

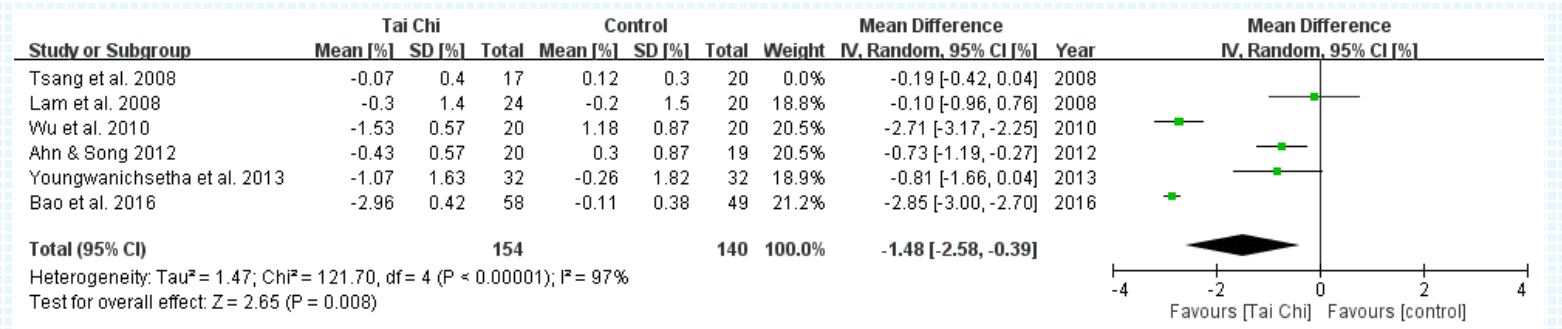

Figure 1 Forest plot of sensitivity analysis of studies on Tai Chi reporting HbAlc

* FBG was measured in six studies, the pooled analysis demonstrated a favorable effect on decreasing FBG (MD: $-1.14 \mathrm{mmol} / \mathrm{L}, 95 \% \mathrm{CI}$ : -1.78 to $-0.50 \mathrm{mmol} / \mathrm{L}, \mathrm{p}<0.01$; Figure 2 ).

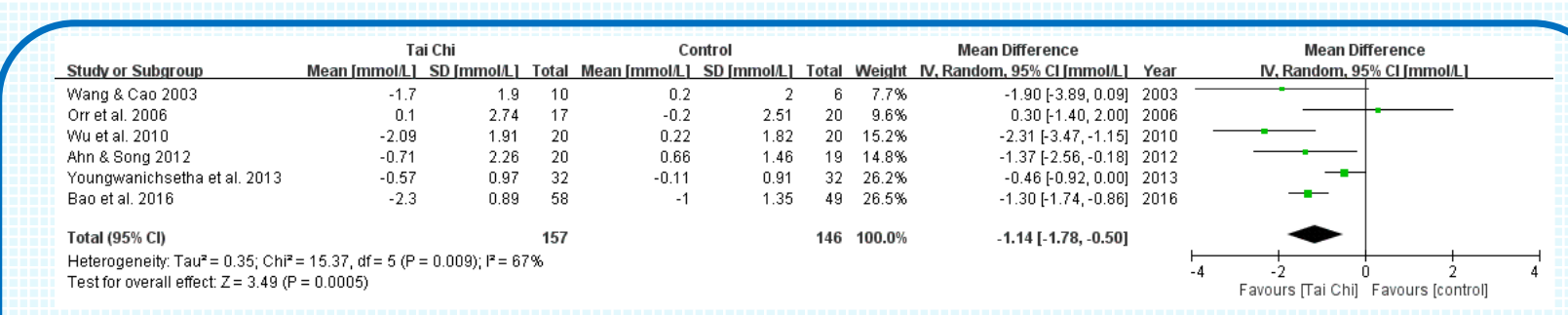

Figure 2 Forest plot of studies on Ba-Duan-Jin reporting FBG

* A pooled analysis of the three studies measured blood pressure indicated a favorable but non-significant effect on decreasing both the systolic blood pressure and the diastolic blood pressure.

* The effects of Tai Chi on QOL were investigated in five studies, the results showed that Tai Chi is effective in improving the physical domain of QOL among T2DM patients (MD=5.92, 95\% CI: 0.68 to $11.16, \mathrm{p}<0.05$; Figure 3 ). The pooled analysis demonstrated a favorable but non-significant effect in improving the mental domain of QOL. However, the effect became statistically significant ( $\mathrm{MD}=6.54,95 \% \mathrm{CI}: 0.77$ to $12.31, \mathrm{p}<0.05)$ after removing the study that recruited participants with good glycemic control (Figure 4).

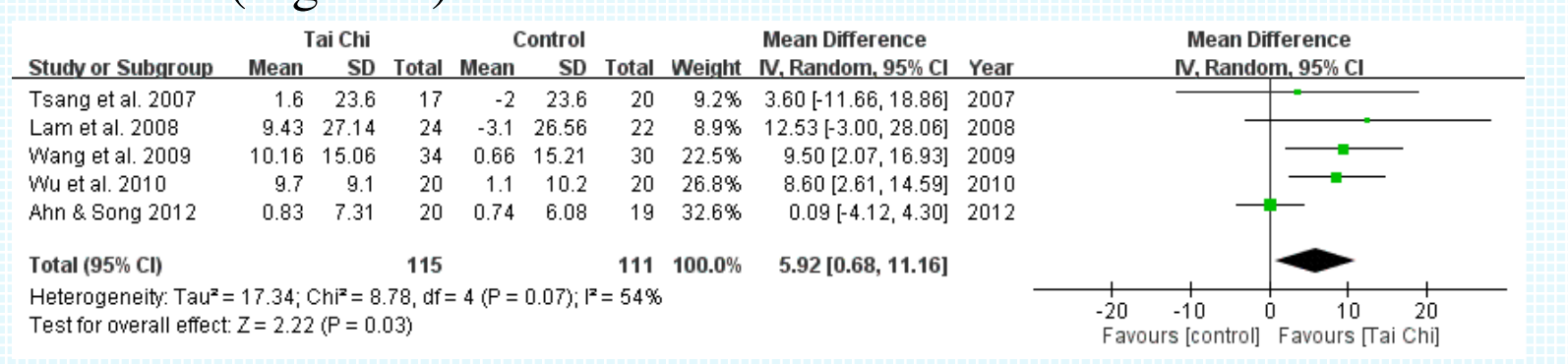

Figure 3 Forest plot of studies on Tai Chi reporting QOL - physical domain

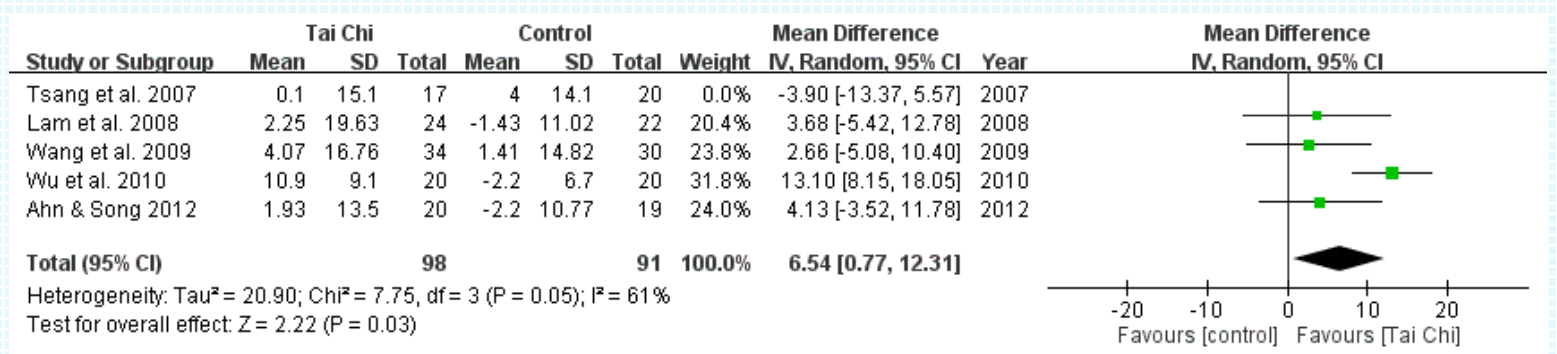

Figure 4 Forest plot of sensitivity analysis of studies on Tai Chi reporting QOL mental domain

* None of the included studies measured depression or the social domain of QOL.

\section{Conclusions}

- This systematic review showed that Tai Chi is effective in reducing HbAlc and FBG, and improving QOL among T2DM patients, especially those whose glycemic control is suboptimal.

- Further studies are worthwhile in order to draw conclusion on the effects of Tai Chi on blood pressure and depression.

- The findings of this systematic review should be interpreted with the consideration of the risks of bias in the included studies and the susceptibility of publication bias of the review itself.

\section{References}

1. International Diabetes Federation. IDF Diabetes Atlas, 7th edn. Brussels, Belgium: International Diabetes Federation; 2015. http://www.diabetesatlas.org 2. Powers MA, Bardsley J, Cypress M, Duker P, Funnell MM, Fischl AH, et al. Diabetes self-management education and support in type 2 diabetes: a joint position statement of the American Diabetes Association, the American Association of Diabetes Educators, and the Academy of Nutrition and Dietetics. Diabetes Educ. 2017;43(1):40-53.

3. Huang J, Yeh M. The Qigong Effect on Blood Glucose Control in People With Type 2 Diabetes: A Systematic Review and Meta-Analysis. Journal of Nursing \& Healthcare Research. 2013;9(3).

4. Lee MS, Chen KW, Choi T, Ernst E. Qigong for type 2 diabetes care: a systematic review. Complement Ther Med. 2009;17(4):236-42.

5. Xin L, Miller YD, Brown WJ. A qualitative review of the role of qigong in the management of diabetes. The Journal of Alternative and Complementary Medicine. 2007;13(4):427-34.

6. Moher D, Liberati A, Tetzlaff J, Altman DG, Prisma Group. Preferred reporting items for systematic reviews and meta-analyses: the PRISMA statement. PLoS med. 2009;6(7):e1000097. 